sein, jedoch reichen die vorliegenden Versuche nicht hin zur Aufstellung einer rationellen Formel.

Mit dem Toluylenoxyd $\mathrm{C}_{14} \mathrm{H}_{12} \mathrm{O}$ isomere Verbindungen entstehen aus den Modificationen des Toluylenalkohols *) beim Kochen mit verdünnter Schwefelsäure. Die eine bildet glasglänzende, bei $132^{\circ}$ schmelzende Prismen, die andere ist ein farbloses 0el. Ausführlicheres hierüber werden wir in einer bald erscheinenden Abhandlung mittheilen und wollen hier nur noch bemerken, dafs das Desoxyanisoin Rossel's **), aus dem Hydranisoïn und Isohydranisoïn durch Kochen mit verdünnter Schwefelsäure dargestellt, deshalb wahrscheinlich auch nicht dem Toluylenoxyd entspricht.

78) Ueber die Benzilsäure oder Diphenylglycolsäure;

von Dr. A. Jena.

Unsere Kenntnifs dieser Säure ist bis jetzt eine sehr beschränkte gewesen. Obgleich sie schon 1838 von L i e big ***) entdeckt wurde, sind später nur von $\mathrm{Zinin} f$ ) und $\mathrm{Ca}$ hours $+f$ ) einige Versuche mit derselben angestellt, welche

*) Schon auf der Naturforscherversammlung zu Innsbruck hat Einer von uns mitgetheilt, dals wenigstens zwei Modificationen des Toluylenalkohols existiren; diese Notiz ist auch in den Berichten der deutschen chemischen Gesellschaft 1869, 610 veröffentlicht.

**) Diese Annalen CLI, 40.

***) Daselbst XXV, 25.

†) Daselbst XXXI, 329.

††) Daselbst LXX, 46. 
uns mit den äufseren Eigenschaften, einigen Salzen und dem Chlorür bekannt gemacht haben. Wahrscheinlich hat die Kostbarkeit dieser Säure und die Schwierigkeit ihrer Darstellung die Chemiker verhindert, sich eingehender mit ihr zu beschäftigen.

Ich habe nur einen Körper gefunden, aus welchem sich die Benzilsäure gewinnen läfst, nämlich das Benzil, aus dem sie beim Erhitzen mit Wasser oder weingeistigem Kali entsteht. Dagegen sind alle Versuche, aus dem Benzoïn mit weingeistigem Kali diese Säure zu erhalten, ohne Erfolg gewesen; wenigstens traten nur so geringe Mengen auf, dafs sie vermuthlich auf eine Verunreinigung des Benzoins mit Spuren Benzil zurückzuführen sind; diese Beobachtung steht in directem Widerspruch mit den Angaben aller Lehrbücher und soll deshalb ausführlicher an einem anderen Orte behandelt werden.

Das Benzil verwandelt sich beim Erhitzen mit Wasser auf $200^{\circ}$ sehr langsam in Benzilsäure :

$$
\underset{\text { Benzil }}{\mathrm{C}_{14} \mathrm{H}_{10} \mathrm{O}_{2}}+\mathrm{H}_{2} \mathrm{O}=\underset{\text { Benzilsäure. }}{\mathrm{C}_{14} \mathrm{H}_{12} \mathrm{O}_{3}}
$$

Nach 6 Stunden war von $1 \mathrm{Grm}$. Benzil noch etwa $3 / 4$ unverändert geblieben, das Uebrige in sehr reine Benzilsäure übergeführt. Die Methode ist daher nicht geeignet zur Darstellung gröfserer Mengen Benzilsäure; man mufs dazu, wie schon Liebig und Zin in thaten, weingeistiges Kali anwenden. Aber auch dabei ist grofse Vorsicht nöthig, wenn sich der Benzilsäure nicht grofse Mengen Benzoësäure beimengen sollen.

Kleine Mengen Benzil ( $1 \mathrm{Grm}$.) verwandeln sich fast vollständig in Benzilsäure beim Erhitzen auf $100^{\circ}$ in zugeschmolzenen Röhren mit wenig Kalihydrat ( $1 / 4$ des Benzils) 
und Weingeist, wie Limpricht und $S \mathrm{chwanert}$ ) schon nachgewiesen haben. Auch bei Anwendung gröfserer Quantitäten Benzil und Ausführung der Operation in einer Digerirflasche nähert sich die Ausbeute der berechneten oft sehr, wenn ein Ueberschufs an Kali vermieden wird. Das Benzil löst sich im weingeistigen Kali mit intensiv violetter Farbe; es tritt darauf die Reaction zuweilen von selbst unter Wärmeentwickelung ein, die sich bis zum Sieden des Weingeistes steigern kann, zuweilen aber erst nach dem Erwärmen mit einer Gasflamme. Nach Beendigung derselben wird so lange erhitzt, bis die Farbe hellgelb geworden ist, Wasser zugefügt und mit Salzsäure gefält. Ist die Benzilsäure nur mit geringen Mengen Benzoësäure verunreinigt, so genügt ein- oder zweimaliges Umkrystallisiren aus heifsem Wasser zur vollständigen Reinigung. Bei Anwesenheit von mehr Benzoësäure digerirt man zweckmälsig den Niederschlag mit einer zur vollständigen Lösung nicht hinreichenden Menge Sodalösung, welche vorzugsweise "die Benzilsäure löst, die dann wieder aus dem Filtrat mit Salzsäure gefällt und aus heifsem Wasser umkrystallisirt wird.

Je mehr Kali im Verhältnifs zum Benzil genommen wird, desto mehr Benzoësäure bildet sich, die zuweilen die Benzilsäure vollständig verdrängt. Beim Lösen der Masse in Wasser bleibt dann ein harziger Körper zurück und beim Fällen des Filtrats mit Salzsäure scheidet sich noch mehr desselben neben Benzoësäure und Benzilsäure aus, die man dem Niederschlage mit kalter Sodalösung entzieht. Durch wiederholtes Lösen des Harzes in Weingeist und Verdunsten der Lösung gewinnt man kleine wasserhelle monoklinometrische Tafeln.

0,384 Grm. lieferten 1,1075 Kohlensäure und 0,2035 Wasser.

*) Berichte d. deutschen chem. Gesellsch. 1869, 134. 


\begin{tabular}{cccc}
\multicolumn{3}{c}{ Berechnet nach der Formel } & Gefunden \\
C & \multicolumn{2}{c}{$\mathrm{C}_{14} \mathrm{H}_{12} \mathrm{O}_{2}$} & 78,7 \\
$\mathrm{H}$ & 168 & 79,3 & 5,8 \\
$\mathrm{O}$ & 12 & 5,6 & - \\
\cline { 2 - 4 } & 32 & 15,1 &
\end{tabular}

Sie besitzen also gleiche Zusammensetzung mit dem Benzoin, aber ganz andere Eigenschaften. Ihr Schmelzpunkt liegt bei $200^{\circ}$; in kaltem Weingeist sind sie sehr schwer und auch in heifsem nicht leicht löslich, und mit Salpetersäure liefern sie kein Benzil.

Diese Verbindung hatte ich in einer vorläufigen Notiz *) Tolanalkohol genannt and vermuthete, da sie immer neben Benzoësäure gefunden wurde, dafs die Zersetzung des Benzils in diesem Falle $n$ ach der Gleichung erfolge

$$
\underset{\text { Benzil }}{2 \mathrm{C}_{14} \mathrm{H}_{10} \mathrm{O}_{2}}+2 \mathrm{H}_{2} \mathrm{O}=\underset{\text { Tolanalkohol }}{\mathrm{C}_{14} \mathrm{H}_{12} \mathrm{O}_{2}}+\underset{\text { Benzoësäure. }}{2 \mathrm{C}_{7} \mathrm{H}_{6} \mathrm{O}_{2}}
$$

In neuerer Zeit habe ich jedoch das Auftreten derselben Verbindung auch beim Erhitzen der Benzilsäure für sich beobachtet, wodurch die Wahrscheinlichkeit obiger Gleichung sehr vermindert wird, und mufs mir vorbehalten, in einer späteren Abhandlung Aufklärung über diesen sogenannten Tolanalkohol zu geben.

Die Benzilsäure bildet kleine monoklinometrische Nadeln, die weifs und atlasglänzend sind, bei $110^{\prime}$ aber schon röthlich werden und bei $150^{\circ}$ schmelzen; in höherer Temperatur tritt tief rothe Färbung ein. Dieselbe Färbung zeigt sich beim Uebergiefsen mit concentrirter Schwefelsäure; Zusatz von Wasser macht sie wieder verschwinden. In Alkohol, Aether und heifsem Wasser ist sie leicht, in kaltem Wasser schwer löslich. Sie besitzt einen bitteren Geschmack.

0,231 Grm. lieferten 0,6252 KoLlensäure und 0,1169 Wasser.

*) Berichte der deutschen chem. Gesellschaft 1869, 384. 
Berechnet nach der Formel

C

\begin{tabular}{rr}
$\mathrm{C}_{14} \mathrm{H}_{12} \mathrm{O}_{3}$ \\
168 & 73,7 \\
12 & 5,3 \\
48 & 21,0 \\
\hline 228 & $100,0$.
\end{tabular}

Gefunden

73,7

5,6

o

Die Angabe früherer Beobachter, dafs der Schmelzpunkt der Benzilsäure bei $120^{\circ}$ liegen solle, hat in allen Lehrbüchern Aufnahme gefunden. Dieses ist aber der Schmelzpunkt der Benzoësäure, die - wie ich schon angeführt habe - in der Regel und häufig in sehr bedeutender Menge neben der Benzilsäure sich bildet und mit Spuren dieser verunreinigt die characteristische rothe Färbung beim Erhitzen und mit concentrirter Schwefelsäure giebt. Es unterliegt daher wohl keinem Zweifel, dafs in den meisten Fällen ein solches Präparat für Benzilsäure gehalten worden ist.

Benzilsaures Baryum, $\left(\mathrm{C}_{14} \mathrm{H}_{11} \mathrm{O}_{3}\right)_{2} \mathrm{Ba}, 6 \mathrm{H}_{2} \mathrm{O}$. - Es wurde durch Lösen der Benzilsäure in Barytwasser und Verdunsten der mit Thierkohle entfärbten Lösung in leicht löslichen weifsen Krystallkrusten gewonnen.

$0,6535 \mathrm{Grm}$. über Schwefelsäme getrocknet verloren bei $150^{\circ}$ 0,0975 Wasser.

0,556 Grm. bei $150^{\circ}$ getrocknet lieferten 0,2194 schwefelsaures Baryum.

Berechnet nach der Formel

$$
\begin{array}{cc}
\left(\mathrm{C}_{14} \mathrm{H}_{11} \mathrm{O}_{3}\right)_{2} \mathrm{Ba}, 6 \mathrm{H}_{2} \mathrm{O}: & \text { Gefunden } \\
15,4 & 15,0
\end{array}
$$

$6 \mathrm{H}_{2} \mathrm{O}$

$\mathrm{Ba}$

$$
\left(\mathrm{C}_{14} \mathrm{H}_{11} \mathrm{O}_{3}\right)_{2} \mathrm{Ba} \text { : }
$$

23,1

23,1 .

Benzilsaures Silber ist ein leicht zersetzbares Salz. Der Niederschlag, welchen Höllenstein in einer neutralisirten Benzilsäurelösung hervorbringt, ist weifs, färbt sich aber bei längerem Stehen und schneller beim Erwärmen mit der Flüssigkeit dunkel. Beim Erhitzen des trockenen Salzes und 
auch schon bei längerem Kochen desselben mit Wasser entsteht Benzophenon.

Der Benzilsäureäther fällt als ein gelbes, nach Pfeffermünze riechendes $0 \mathrm{el}$, wenn die mit Chlorwasserstoff gesättigte weingeistige Lösung der Benzilsäure mit Wasser vermischt wird. Er krystallisirt nicht und zersetzt sich bei der Destillation.

Bei mehrstündigem Erhitzen der Benzilsäure auf $180^{\circ}$ im Oelbade entsteht eine tiefrothe Flüssigkeit, die beim Erkalten zu einer amorphen Masse erstarrt. In Weingeist ist sie leicht löslich und beim Verdunsten der weingeistigen Lösung scheiden sich mehrere krystallisirte Verbindungen ab, von welchen ich nur Dibenzilsäure $\mathrm{C}_{28} \mathrm{H}_{22} \mathrm{O}_{5}$, sogenannten Tolanalkohol $\mathrm{C}_{14} \mathrm{H}_{12} \mathrm{O}_{2}$ und Benzophenon $\mathrm{C}_{13} \mathrm{H}_{10} \mathrm{O}$ anführen will. Ich habe die hier vor sich gehende complicirte Reaction noch nicht vollständig aufklären können und werde diesen Theil der Untersuchung erst später liefern.

Eine ähnliche Zersetzung scheint die Benzilsäure beim Erhitzen mit Salzsäure auf $150^{\circ}$ zu erleiden; es entsteht ein rothes $0 \mathrm{el}$, in welchem Dibenzilsäure nachgewiesen wurde.

Bei der Destillation der Benzilsäure geht ein rothbraunes empyreumatisch riechendes $0 \mathrm{el}$ über, das mit Wasserdämpfen überdestillirt werden kann, für sich erhitzt aber bei einer Temperatur von 270 bis $280^{\circ}$ verkohlt.

Die Benzilsäure verändert sich nicht bei dreistündigem Erhitzen mit weingeistigem Kali auf $150^{\circ}$.

Das benzilsaure Baryum zerlegt sich bei der Destillation mit $1 / 10$ Natronkalk in kohlensaures Salz und Benzhydrol :

$$
\underset{\text { Benzilsäure }}{\mathrm{C}_{14} \mathrm{H}_{12} \mathrm{O}_{3}}=\mathrm{CO}_{2}+\underset{\text { Benzhydrol. }}{\mathrm{C}_{13} \mathrm{H}_{12} \mathrm{O}}
$$

Das Destillat war ein gelbes Oel, das in sehr niedriger Temperatur krystallinisch erstarrte. Der Siedepunkt wurde 
bei $295^{\circ}$ beobachtet; Linne mann*) giebt den des Benzhydrols zu $297^{\circ}$ an. Die bei Winterkälte abgeprefsten Krystalle schmolzen bei $60^{\circ}$; Linnemann giebt in der citirten Ahhandlung S. 8 den Schmelzpunkt $67^{\circ}, 5$ bis $68^{\circ}$, dagegen S. 28 den Schmelzpunkt $57^{\circ}, 5$ bis $58^{\circ}$ an. Die einmal geschmolzenen Krystalle wurden erst nach sehr langem Stehen in der Kälte wieder fest.

0,3675 Grm. lieferten 1,154 Kohlensïure und 0,208 Wasser.

Berechnet nach der Formel

\begin{tabular}{lrrr} 
& \multicolumn{2}{c}{$\mathrm{C}_{13} \mathrm{H}_{12} \mathrm{O}$} & Gefunden \\
\cline { 2 - 3 } & 156 & 84,8 & 85,6 \\
$\mathrm{H}$ & 12 & 6,5 & 6,3 \\
& 16 & 8,7 & -
\end{tabular}

Diese Analyse, welche leider nur mit der flüssigen Substanz, nicht mil Krystallen ausgeführt werden konnte, läfst auf eine Beimengung von Benzophenon schliefsen, das durch den oxydirenden Einfluls des Natronkalks aus dem Benzhydrol entstanden ist. Eine Reinigung durch Destillation oder Krystallisation liefs sich mit der kleinen mir zu Gebote stehenden Menge nicht ausführen; ich versuchte deshalb, um noch einen Anhaltspunkt für das Vorhandensein des Benzhydrols zu haben, nach Linn emann's Vorschrift den Bernsteinsäureäther desselben darzustellen, und erhielt auch die von Jenem beschriebenen kleinen Schüppchen, welche bei 137 bis $138^{\circ}$ schmolzen (L in n e m a n n giebt den Schmelzpunkt $141^{\circ}$ an). Das Aüftrelen des Benzhydrols bei Destillation des benzilsauren Baryums ist hiermit bewiesen.

Oxydirende Substanzen, z. B. Chromsäure, verwandeln die Benzilsäure in Kohlensäure, Wasser und Benzophenon :

$$
\underset{\text { Benzilsture }}{\mathrm{C}_{14} \mathrm{H}_{12} \mathrm{O}_{3}}+\mathrm{O}=\underset{\text { Benzophenon. }}{\mathrm{C}_{13} \mathrm{H}_{10} \mathrm{O}}+\mathrm{CO}_{2}+\mathrm{H}_{2} \mathrm{O}
$$

*) Diese Annalen CXXXIII, 8. 
Auch benzilsaures Silber liefert bei trockener Destillation diese Verbindung. - Benzilsäure (1 Thl.) wird mit chromsaurem Kalium (2 Thl.), Schwefelsäure (3 Thl.) und Wasser (3 Thl.) mehrere Stunden im Wasserbade erwärmt, der Rückstand nach dem Erkalten mit Aether ausgeschüttelt und das beim Verdampfen des Aethers bleibende Benzophenon durch Destillation gereinigt. Es ist wegen des heftigen Stofsens nicht rathsam, das Benzophenon nach vollendeter Oxydation mit Wasserdämpfen abzudestilliren.

Das Benzophenon siedete bei $304^{\circ}$ (L inn e mann [a. a. 0.] giebt $295^{\circ}$, Chancel [diese Annalen LXXII, 279] $315^{\circ}$ als Siedepunkt an), war vollkommen farblos und erstarrte in der Winterkälte. Die Krystalle schmolzen bei $44^{\circ}$.

1. 0,293 Grm. lieferten 0,9208 Kohlensäure und 0,1517 Wasser

2. 0,244 Gr'm. lieferten 0,7662 Kohlensäure und 0,1236 Wasser.

Berechnet nach der Formel

\begin{tabular}{rrrrr} 
& \multicolumn{2}{c}{$\mathrm{C}_{14} \mathrm{H}_{18} \mathrm{O}$} & \multicolumn{1}{c}{1.} & \multicolumn{1}{c}{ C. } \\
$\mathrm{C}$ & $\widehat{168}$ & 85,7 & 85,7 & 85,6 \\
$\mathrm{H}$ & 13 & 5,4 & 5,7 & 5,6 \\
$\mathrm{O}$ & 16 & 8,9 & - & -
\end{tabular}

Die Benzilsäure erleidet bei Behandlung mit Zink und Salzsäure oder mit Natriumamalgam keine Veränderung. Jedoch bei vierstündigem Erhitzen auf $150^{\circ}$ mit Jodwasserstoffsäure (1270 Siedepunkt) wird sie durch Abgabe von 1 At. 0 verwandelt in Diphenylessigsäure, $\mathrm{C}_{14} \mathrm{H}_{12} \mathrm{O}_{2}$. - Erhitzt man viel länger oder auf eine höhere Temperatur, so wird ein nicht näher untersuchtes $0 \mathrm{el}$ gebildet. - Der Inhalt der Röhren wird mit Wasser vermischt, das Ungelöste in Soda aufgenommen, aus dieser Lösung mit Salzsäure wieder gefällt und der Niederschlag aus heifsem Wasser umkrystallisirt.

Aus heifsem Wasser krystallisirt die Diphenylessigsäure in Nadeln, welche Aehnlichkeit mit der Benzilsäure haben, aus Weingeist können lange flache Blätter gewonnen werden; 
in kaltem Wasser ist sie schwer, in heifsem Wasser, Weingeist und Aether leicht löslich. Sie schmilzt bei $146^{\circ}$, giebt bei stärkerem Erhitzen ein geringes Sublimat und färbt sich dabei nicht roth. Auch mit concentrirter Schwefelsäure tritt keine rothe, sondern beim Erwärmen eine grünlich-gelbe Färbung ein.

1. 0,1818 Grm. lieferten 0,5255 Kohlensïure und 0,0965 Wasser.

2. 0,1791 Grm. lieferten 0,5186 Kohlensäure und 0,0921 Wasser.

\begin{tabular}{crrrrr} 
& \multicolumn{2}{c}{ Berechnet nach der Formel } & \multicolumn{2}{c}{ Gefunden } \\
$\mathrm{C}$ & \multicolumn{2}{c}{$\mathrm{C}_{14} \mathrm{H}_{12} \mathrm{O}_{2}$} & \multicolumn{1}{c}{$\mathbf{2 .}$} & 2. \\
$\mathrm{H}$ & 168 & 79,3 & 78,8 & 78,9 \\
$\mathrm{O}$ & 12 & 5,6 & 5,8 & 5,7 \\
& 32 & 15,1 & - & - \\
\cline { 2 - 5 } & 212 & $100,0$. & &
\end{tabular}

Diphenylessigsaures Baryum, $\left(\mathrm{C}_{14} \mathrm{H}_{11} \mathrm{O}_{2}\right)_{2} \mathrm{Ba}, 2 \mathrm{H}_{2} \mathrm{O}$. In Wasser leicht lösliche, zu Büscheln vereinigte allasglänzende Nadeln, die erst über $300^{\circ}$ schmelzen.

0,471 Grm., Iufttrocken, lieferten bei $200^{\circ} 0,0300$ Wasser.

0,438 Grm, bei $200^{\circ}$ getrocknet, lieferten 0,1842 schwefelsaures Baryum.

$\begin{array}{ccc} & \text { Berechnet nach der Formel } \\ & \left(\mathrm{C}_{14} \mathrm{H}_{11} \mathrm{O}_{2}\right)_{2} \mathrm{Ba}, 2 \mathrm{H}_{2} \mathrm{O}: & \text { Gefunden } \\ 2 \mathrm{H}_{2} \mathrm{O} & 6,2 & 6,3 \\ & \left(\mathrm{C}_{14} \mathrm{H}_{11} \mathrm{O}_{2}\right)_{2} \mathrm{Ba}: & \\ \mathrm{Ba} & 24,5 & 24,6 .\end{array}$

Diphenylessigsaures Silber, $\mathrm{C}_{14} \mathrm{H}_{11} \mathrm{AgO} \mathrm{O}_{2}, 2 \mathrm{H}_{2} \mathrm{O}$. - Weifser, aus feinen kleinen Nadeln bestehender Niederschlag, den salpetersaures Silber in einer neutralen Lòsung der Diphenylessigsäure hervorbringt. Am Lichte schwärzt er sich.

$0,724 \mathrm{Grm}$. lieferten bei $100^{\circ} 0,0726$ Wasser.

0,698 Grm. lieferten 0,2813 Chlorsilber.

Berechnet nach der Formel

$\begin{array}{ccc} & \mathrm{C}_{14} \mathrm{H}_{11} \mathrm{AgO}_{2}, 2 \mathrm{H}_{2} \mathrm{O} & \text { Gefunden } \\ 2 \mathrm{H}_{2} \mathrm{O} & 10,1 & 10,0 \\ \mathrm{Ag} & 30,4 & 30,3 .\end{array}$


Diphenylsumpfgas, $\mathrm{C}_{13} \mathrm{H}_{12}$. - Das Baryumsalz der Diphenylessigsäure liefert bei der Destillation mit wenig Natronkalk ein 0el, das in sehr niedriger Temperatur krystallisirte. Es wurde in Weingeist gelöst, die Lösung mit Thierkohle behandelt, verdunstet und der Rückstand in einer Kältemischung zum Erstarren gebracht.

Klinorhombische Prismen, äufserst angenehm nach Orangen riechend, bei $26^{\circ}, 4$ schmelzend, in Aether und Weingeist leicht löslich. Die ätherische Lösung giebt mit Brom nach einiger Zeit einen Niederschlag.

$0,2805 \mathrm{Grm}$. lieferten 0,7043 Kohlensäure und 0,1395 Wasser. Berechnet nach der Formel

\begin{tabular}{lrrr} 
& \multicolumn{2}{c}{$\mathrm{C}_{13} \mathrm{H}_{12}$} & Gefunden \\
\cline { 2 - 4 } $\mathrm{C}$ & 156 & 92,8 & 92,1 \\
$\mathrm{H}$ & 12 & 7,2 & 7,4 \\
\cline { 2 - 4 } & 168 & $100,0$. &
\end{tabular}

Die Diphenylessigsäure wird sehr schwierig von Chromsäure angegriffen. Nach 12 siündiger Einwirkung von chromsaurem Kalium und Schwefelsäure im Wasserbade war der gröfste Theil der Säure unverändert geblieben, dabei aber ein Harz entstanden, das sich schwer reinigen, namentlich von Chromoxyd trennen liefs. Durch sehr oft wiederholtes Lösen und Krystallisirenlassen wurden Krystalle von Benzophenon (Schmelzpunkt 45 ${ }^{\circ}$ ) gewonnen, das sich nach der Gleichung

$$
\underset{\text { Diphenylessigsäure }}{\mathrm{C}_{14} \mathrm{H}_{12} \mathrm{O}_{2}}+\underset{\text { Benzophenon }}{\mathrm{O}_{2}}=\underset{\mathrm{CO}_{2}}{\mathrm{C}_{13} \mathrm{H}_{10} \mathrm{O}}+\mathrm{H}_{2} \mathrm{O}
$$

gebildet hat.

Die Unwandlung des Benzils in Benzilsäure durch Aufnahme von Wasser, wenn es mit Wasser oder mit weingeistigem Kali erhitzt wird, spricht sehr zu Gunsten der Ansicht, dafs es das Anhydrid der Benzilsäure ist. Ich versuchte jetzt auch aus der Benzilsäure das Benzil durch Behandlung mit Phosphorsäureanhydrid darzustellen. - Beim 
Mischen der trockenen Säure in kleinen Portionen mit dem Phosphorsäureanhydrid, welches sich in einem weiten Probirrohr befand, färbte sich die Masse unter starker Wärmeentwickelung violett, und bildete, als zuletzt noch gelinde erwärmt worden war, nach dem Erkalten eine harte Masse. Diese wurde mit Wasser behandelt, der in Wasser unlösliche Rückstand in Weingeist gelöst, die Lösung mit Thierkohle digerirt, filtrirt und verdunstet. Es schieden sich zuerst Krystalle der Dibenzilsäure, $\mathrm{C}_{28} \mathrm{H}_{22} \mathrm{O}_{5}$, ab und zuletzt blieb ein dickflüssiges, auch nach Monaten nicht krystallisirendes Oel. Diese Eigenschaft nicht zu krystallisiren scheint mir noch kein Beweis, dafs der Rückstand nicht Benzil sei; denn ich habe bei diesem Körper zu oft beobachtet, dafs er in nicht ganz reinem Zustande genau dasselbe Verhalten zeigt und sich dann als dickes Oel statt in Krystallen aus seiner Lösung abscheidet. Zwei Versuche machen mir wenigstens das Vorhandensein von Benzil in diesem Oel sehr wahrscheinlich. Es wurde aus demselben durch Erhitzen mit Wasser auf $200^{\circ}$ und durch Kochen mit weingeistigem Kali reine Benzilsäure dargestellt.

Noch einige Versuche, die ich mit dem Benzil angestellt habe, mögen hier kurz erwähnt werden.

Das mit Natronkalk gemengte Benzil giebt beim Erhitzen ein aus Benzol und Benzophenon bestehendes Destillat; das Benzophenon wurde vollkommen gereinigt und in Krystalle übergeführt.

Wird das Benzil mit einer weingeistigen Lösung von Kaliumsulfhydrat in zugeschmolzenen Röhren auf $120^{\circ}$ erhitzt, so verwandelt es sich in Benzoïn und Toluylenoxyd. Beide Verbindungen wurden in so reinem Zustande gewonnen, dafs ihre Identität durch Schmelzpunkt und Elementaranalyse festgestellt werden konnte. Die folgenden Gleichungen veranschaulichen diese Metamorphose : 


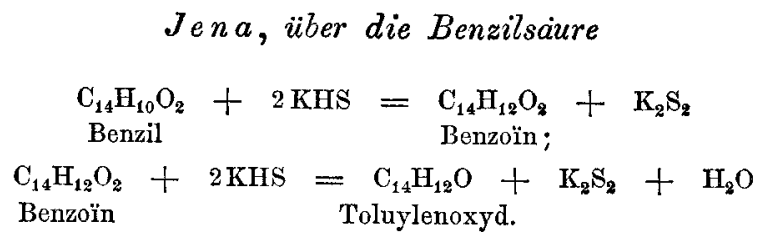

Schon mehrere Chemiker haben versucht, eine rationelle Formel für die Benzilsäure aufzustellen. Die von Grima ux*) gegebene<smiles>OCCCCCCCCCCCCO</smiles>

der sich die von Kekulé $\left.{ }^{* *}\right)$

$$
\begin{aligned}
& \mathrm{C}_{6} \mathrm{H}_{5} \cdot \mathrm{C}-\mathrm{OH} \\
& \mathrm{C}_{6} \mathrm{H}_{5} \cdot \mathrm{C}-\mathrm{OH}
\end{aligned}
$$

anschliefst, läfst ihre nahe Beziehung zur Glycolsäure nicht erkennen. Lagert man dagegen die beiden Atome Phenyl an ein und dasselbe Kohlenstoffatom, wie Oppenheim ***) und Städeler t) vorgeschlagen haben :

$$
\begin{aligned}
& \mathrm{C}\left(\mathrm{C}_{6} \mathrm{H}_{5}\right)_{2} \cdot \mathrm{OH} \\
& \mathrm{CO}, \mathrm{OH}
\end{aligned}
$$

und wie auch die nicht ganz glürklich gewählte Formel von Limpricht und $S c h w a n e r t+$ ) ausdrücken soll :

$$
\left(\mathrm{C}_{6} \mathrm{H}_{5}\right)_{2}\left\{\begin{array}{l}
\mathrm{COHO} \\
\mathrm{COH},
\end{array}\right.
$$

so wird damit der Ansicht, die Benzilsäure als Diphenylglycolsäure zu betrachten, ein nicht mifszuverstehender Ausdruck gegeben. Die Benzilsäure und die ihr nahestehenden Verbindungen bekommen dann die folgenden Formeln :

\footnotetext{
*) Dictionnaire de chimie par Wurtz I, 552 .

**) Berichte der deutschen chem. Gesellschaft 1869, 611.

***) Daselbst 1869, 281.

f) Diese Annalen CLI, 45.

†) Berichte der deutschen chem. Gesellschaft 1869, 135.
} 


$$
\begin{aligned}
& \mathrm{C}\left(\mathrm{C}_{6} \mathrm{H}_{5}\right)_{2} \mathrm{HO} \\
& \mathrm{COHO}
\end{aligned}
$$

Benzilsäure,

Diphenylglycolsäure

$$
\begin{aligned}
& \mathrm{C}\left(\mathrm{C}_{6} \mathrm{H}_{5}\right)_{2} \mathrm{H} \\
& \mathrm{COHO}
\end{aligned}
$$

Diphenylessigsäure.

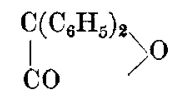

\section{9) Ueber das Benzoïn;}

von A. Jena und H. Limpricht.

Mit der Untersuchung verschiedener Glieder der Toluylengruppe beschäftigt, konnten wir es nicht umgehen, mehrere von anderen Chemikern gemachte Angaben über das Benzoïn einer nochmaligen Prüfung zu unterziehen. Unsere Resultate, die nur zum Theil mit jenen Angaben übereinstimmen, theilen wir im Folgenden mit.

Hinsichtlich des Schmelzpunktes schon weichen unsere Beobachtungen ab. Derselbe liegt nicht bei $120^{\circ}$, wie zuerst Liebig und Wöhler und später alle Chemiker angeben, sondern bei $137^{\circ}$. Wir haben zur Feststellung dieser Thatsache $\mathbf{z u}$ verschiedenen Zeiten von uns selbst aus Bittermandelöl mit Cyankalium, ferner aus Toluylenalkohol mit Salpetersäure dargestelltes und endlich von Trommsdorf in Erfurt und Marquardt in Bonn bezogenes Benzoïn, nachdem wir uns nach mehrmaligem Umkrystallisiren durch die Elementaranalyse von seiner Reinheit überzeugt hatten, geprüf, und immer den Schmelzpunkt $137^{\circ}$ beobachtet.

Das Benzoïn geht bei der Destillation fast unverändert über, nur zuletzt tritt Bittermandelöl in geringer Menge auf; 